Scientific Paper

\title{
Trend and prediction of COVID-19 outbreak in Iran: SEIR and ANFIS model
}

\author{
Sajad SHAFIEKHANI ${ }^{a, b, c}$, Touraj Harati KHALILABADd, Sima RAFIEI ${ }^{e}$, Vahid SADEGHI ${ }^{f}$, Amir Homayoun JAFARI ${ }^{a, b}$, \\ Nematollah GHEIBIg, ${ }^{*}$ \\ ${ }^{a}$ Departments of Biomedical Engineering, School of Medicine, Tehran University of Medical Sciences, Tehran, Iran \\ ${ }^{b}$ Research Center for Biomedical Technologies and Robotics, Tehran, Iran \\ 'Students' Scientific Research Center, Tehran University of Medical Sciences, Tehran, Iran \\ ${ }^{d}$ Department of Health Economics, School of Health Management and Information Sciences, Iran University of Medical \\ Sciences, Tehran, Iran \\ ${ }^{e}$ Department of Healthcare Management, School of Health, Qazvin University of Medical Sciences, Qazvin, Iran \\ ${ }^{f}$ Departments of Biomedical Engineering, School of Medicine, Isfahan University of Medical Sciences, Isfahan, Iran \\ ${ }^{g}$ Cellular and Molecular Research Center, Research Institute for Prevention of Non-Communicable Diseases, Qazvin \\ University of Medical Sciences, Qazvin, Iran \\ *E-mail address: gheibi_n@yahoo.com
}

\begin{abstract}
Background: Mathematical and predictive modeling approaches can be used in COVID-19 crisis to forecast the trend of new cases for healthcare management purposes. Given the COVID-19 disease pandemic, the prediction of the epidemic trend of this disease is so important.

Methods: We constructed an SEIR (Susceptible-Exposed-Infected-Recovered) model on the COVID-19 outbreak in Iran. We estimated model parameters by the data on notified cases in Iran in the time window 1/22/2020 - 20/7/2021. Global sensitivity analysis is performed to determine the correlation between epidemiological variables and SEIR model parameters and to assess SEIR model robustness against perturbation to parameters. We Combined Adaptive NeuroFuzzy Inference System (ANFIS) as a rigorous time series prediction approach with the SEIR model to predict the trend of COVID-19 new cases under two different scenarios including social distance and non-social distance.

Results: The SEIR and ANFIS model predicted new cases of COVID-19 for the period February 7, 2021, till August 7, 2021. Model predictions in the non-social distancing scenario indicate that the corona epidemic in Iran may recur as an immortal oscillation and Iran may undergo a recurrence of the third peak.

Conclusion: Combining parametrized SEIR model and ANFIS is effective in predicting the trend of COVID-19 new cases in Iran.
\end{abstract}

Key words: coronavirus disease 2019 (COVID-19); Susceptible-Exposed-Infectious-Recovered (SEIR); Adaptive Neuro-Fuzzy Inference System (ANFIS), global sensitivity analysis.

\section{Introduction}

In December 2019, a viral outbreak was reported in Wuhan, China. ${ }^{1}$ The cause of this disease was a new and genetically modified virus from the family of coronaviruses called SARS 2$\mathrm{CoV}$, which was named COVID-19 disease. Unfortunately, due to the very high transmission rate, the virus spread rapidly throughout the world and infected almost all countries of the world in a short time (less than four months), ${ }^{2,3}$

According to recent reports more than 27000000 and 890000 people around the world have been infected and died due to the virus until 10 September 2020, respectively. ${ }^{4}$ COVID-19 affects the respiratory system of the body and is spreading catastrophically. The main symptoms and clinical signs in the cases include fever, cough, dyspnea, chest pain, lightheadedness, nausea, vomiting, myalgia, diarrhea, and hemoptysis. ${ }^{\mathbf{5}, \mathbf{6}}$ In Iran, the first case of COVID-19 was reported on 19 February 2020 in Qom city. Then the disease has spread rapidly in neighboring provinces.

However, due to the limited amount of information on the pathogenicity of the SARS-CoV-2 virus as well as methods of control and treatment of this disease, currently, the most important way to deal with COVID-19 disease is to prevent the spread of the virus. ${ }^{7}$ Given the COVID-19 disease pandemic, which affects all-important economic, political, social, and even military aspects of all countries in the world, the issue of predicting the trend of this disease in the country is so important. $^{8}$ 
Since the beginning of the COVID-19 epidemic, several studies have examined and predicted the disease. ${ }^{\mathbf{8}, 9}$ The results of most predictions showed an increase in the number of cases to some point in time and then a decrease in the number of cases. Implementing social distancing programs, quarantine, and raising public awareness were among the reasons for the decline in the number of cases worldwide, including in Iran. However, on the other hand, the trend of cases in Iran has become different from other countries. In Iran, the implementation of the social distancing program failed due to the holding of religious, traditional ceremonies, and the instability of the people to observe health points. Therefore, after reducing the number of cases, the disease started to increase again. ${ }^{\mathbf{1 0}}$

Corona in Iran had three peaks, the third of which includes a large population. At the time of writing this manuscript, Iran is at the beginning of the ancient holiday of Nowruz, and there is a high probability that the third peak will be repeated and many people may be exposed to the SARS 2-CoV virus. Predicting the COVID-19 trend in Iran, and informing health planners and policymakers about the number of cases in the future, can play an important role in controlling the disease and providing health services in the country. Therefore, this study predicts COVID19 in Iran by combining the SEIR model and the ANFIS model. SEIR model widely has been used to capture dynamics of infected diseases. ${ }^{2,11}$ Also, ANFIS is a time series predictive approach that can be used to forecast the trend of COVID-19. ${ }^{12-14}$ Since the pattern of the SARS 2-CoV virus spread in Iranian society is not uniform, the use of time series prediction methods such as the ANFIS model along with SEIR modeling can help predict the trend of the COVID-19 disease.

\section{Material and Methods}

A flowchart of the SEIR model is provided in Figure 1. The SEIR model of this study is based on the mechanistic interaction of four sub-classes namely, Susceptible (S), Exposed (E), Infected (I), and Recovered (R). We used four ordinary differential equations (ODEs) to simulate the dynamics of the population of each compartment. In the following, the formulation of ODEs and a description of epidemiological parameters related to mechanistic interactions of different compartments are given.

\section{Susceptible $(S)$}

$\frac{d S}{d t}=\beta \delta-\beta \frac{S I}{N}-\mu S$

According to Equation 1, the population of susceptible compartment increased due to rate $\beta \delta$. The recruitment rate $\beta$ is a function of time which is the sum of a constant rate $\alpha_{0}$ with $n_{i}$ Gaussian functions with coefficients $\alpha_{\mathrm{i}}, \mathrm{i}=1: n_{i}$. The parameters of Gaussian functions are provided in Table 1. Due to the oscillating dynamics in the confirmed cases of COVID-19 in Iran, the susceptible population is recruited with a timevarying function:

$\beta=\alpha_{0}+\sum_{i=1}^{n_{i}} \alpha_{i} e^{-\left(\frac{t-t_{i}}{\sigma_{i}}\right)^{2}}$

Eq. 2

The susceptible population decreased due to contact/interaction with the infected population with rate $\beta \frac{S I}{N}$, and due to natural death with exponential rate $\mu$, that $N=S+I+R$.

\section{Exposed $(E)$}

$\frac{d E}{d t}=\beta \frac{S I}{N}-k E-\mu E$

Equation 3 describes the dynamics of the population of the exposed compartment. The population of exposed compartment increased by contact/interaction of infected patients with susceptible individuals with a rate $\beta \frac{S I}{N}$. Exposed populations become infected after the disease incubation period $\frac{1}{k}$ and the population of exposed compartment declines by natural death rate $\mu$.

\section{Infected $(I)$}

$\frac{d I}{d t}=k E-\theta I-\mu I$

Eq. 4

As formulated in Equation 4, the population of the infected patients increased due to the entry of exposed individuals to the infected compartment after the incubation period $1 / k$. The population in this compartment decreased due to recovery rate $\Theta$ and natural death rate $\mu$.

\section{Recovered $(\boldsymbol{R})$}

According to Equation 5, the population of recovered compartment increased due to the recovery of infected patients with rate $\Theta$ and decreased due to natural death with rate $\mu$.

$\frac{d R}{d t}=\theta I-\mu R$

Eq. 5

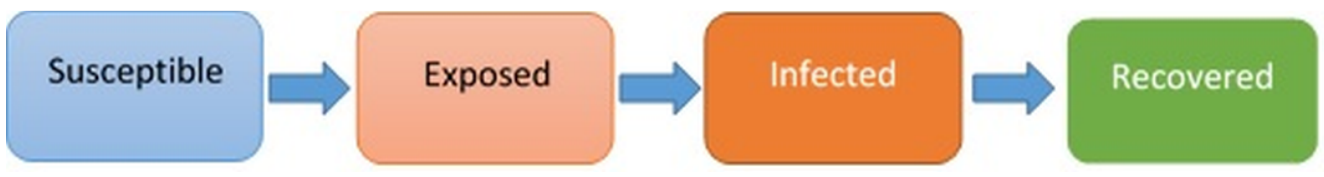

Figure 1. Flow chart of the SEIR model. 


\section{Data}

We obtained data on the daily notified COVID-19 cases in the time window 1/22/2020-2/7/2021 that is reported by the World Health Organization (WHO). We parametrized the SEIR model by this data to fit the dynamics of COVID-19 cases to the daily notified cases by WHO. The dynamics generated by parametrized SEIR model are given as input to the ANFIS model to predict disease progression for the next six months under different scenarios. Actually, we used the predicted dynamics of the SEIR model in the time window 1/22/2020 - 2/07/2021 as data to train and test the ANFIS model. For this aim, we designed the following data set:

$$
\left[\begin{array}{ccc}
X(1) x(2) & x(3) & X(4) \\
\vdots & \vdots & \vdots \\
X(372) x(373) & X(374) & X(375)
\end{array}\right]
$$

We used the first three elements of $i^{\text {th }}, i \in$ [1:372], row as inputs of ANFIS model to predict the fourth element of that row. $X(t)$ represents the population of infected cases in Iran in time point (day) $t$. Therefore, $X(l)=\operatorname{ANFIS}(X(i), X(j), X(k)), i \in$ [1:372], $j \in[2: 373], k \in[3: 374], l \in[4: 375]$.

In the next sections, we describe the setting of the ANFIS model.

\section{SEIR model fitting}

Epidemiological parameters of our SEIR model are estimated by fitting the model on daily notified COVID-19 cases in Iran. Model calibration is performed by fminsearch command in MATLAB 2019b. Also, Akaike Information Criteria (AIC) is used to select the simplest form of model. ${ }^{15}$ AIC analysis used to find the minimum number of Gaussian functions (minimum $\left.n_{i}\right)$. AIC formulation id given by

$A I C=n \ln \left(\frac{S S R}{n}\right)+\frac{2(K+1) n}{n-K-2}$

Eq. 6

Where $n$ is the number of data points, SSR is the fitting error, and $K=5+3 n_{i}$ is the total number of parameters (each Gaussian function has 3 parameters and we have $n_{i}$ Gaussian functions and 5 fixed parameters).
Normalized root-mean-square error (NRMSE) is defined as a cost function of the optimization algorithm. The deviation between the infected population predicted by the model and reported daily cases in Iran is used as an error of the optimization algorithm.

\section{Global sensitivity analysis}

In this section, we assessed the robustness of the SEIR model against parameter fluctuations. We used the partial rank correlation coefficient (PRCC) method to compute the correlation between epidemiological variables (S, E, I, R) with respect to the model parameters. Following sensitivity analysis developed in $\mathbf{1 6}$, we assigned uniform distribution for all parameters of the model (in the range of one-half to twice its value in Table 1) and generate 1000 samples from these distributions using Latin hypercube sampling (LHS). Then we evaluate the model by these samples and compute the PRCC values and the corresponding p-values (significance level) with respect to the averaged dynamic of all epidemiological variables.

\section{ANFIS model}

In this study, we generated a Fuzzy Inference System (FIS) using the fuzzy c-means (FCM) clustering method to extract a set of fuzzy rules that models the behavior and trend of COVID-19 cases in Iran. FCM rule extraction determines the number of fuzzy rules and estimates the parameters of membership functions for the antecedents and consequents of rules. As shown in Figure 2, the trained ANFIS model uses the population of COVID-19 cases at days $t-2, t-1, t$ to predict the population of COVID-19 cases at day $t+1$. The training and testing of the ANFIS model are performed by data of daily notified COVID-19 cases in Iran. The data is partitioned into two training ( $75 \%$ of data) and test (25\% of data) sections to train and validate the ANFIS model.

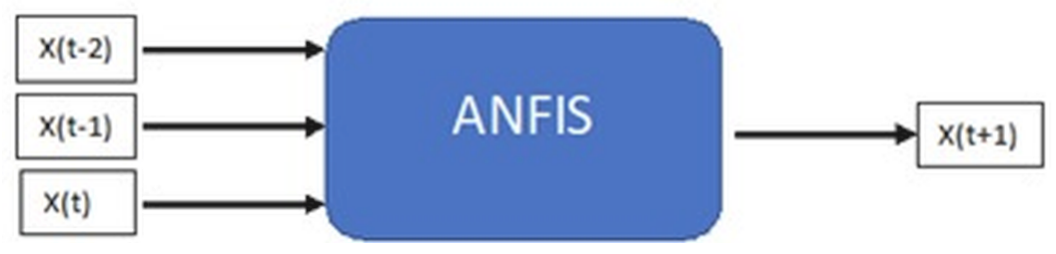

Figure 2. The inputs and output of the predictive ANFIS model. ANFIS model predicts the population of new cases of COVID-19 by the population of COVID-19 cases of three days ago. 


\section{Results}

First, we parametrized the SEIR model based on data of daily notified COVID-19 cases. The estimated parameters are provided in Table 1. As shown in Figure 3, the parametrized SEIR model fitted to the daily notified cases of COVID-19 in Iran. As shown in Figure 4, we computed the value of AIC by setting $n_{i}=1: 12$, and we found that $n_{i}=9$ is the best setting. Also, as depicted in Figure 5, the ANFIS model is calibrated by the training data set (75\% of data) and is validated by the test dataset (25\% of data). We showed the regression of ANFIS model outputs in Figure 6 to show ANFIS model predictive power.

Table 1. Epidemiological parameters of the SEIR model.

\begin{tabular}{|c|c|c|}
\hline Parameter & Value (Range) & Reference \\
\hline$\delta$ & Average recruitment rate & Estimated \\
\hline$\beta$ & The transmission rate of infection & Estimated \\
\hline $1 / \mu$ & Average life expectancy & 17 \\
\hline $1 / k$ & COVID-19 incubation period & 18 \\
\hline$\theta$ & The probability that an infected patient will be recovered & 19 \\
\hline$\alpha_{i=0: 9}$ & $\begin{array}{l}\alpha_{0}=0.238, \alpha_{1}=18.071, \alpha_{2}=213.901, \alpha_{3}=14.427, \alpha_{4}=25.629 \\
\alpha_{5}=89.301, \alpha_{6}=9.284, \alpha_{7}=0.319, \alpha_{8}=120.789, \alpha_{9}=118.104\end{array}$ & Estimated \\
\hline$t_{i=1: 9}$ & $\begin{array}{c}t_{1}=70.503, t_{2}=164.248, t_{3}=81.460, t_{4}=139.844 \\
t_{5}=307.408, t_{6}=202.275, t_{7}=2220.826, t_{8}=420.870, t_{9}=307.900\end{array}$ & Estimated \\
\hline$\sigma_{i=1: 9}$ & $\begin{array}{c}\sigma_{1}=1.941, \sigma_{2}=0.153, \sigma_{3}=9.702, \sigma_{4}=40.545 \\
\sigma_{5}=70.095, \sigma_{6}=25.472, \sigma_{7}=193.890, \sigma_{8}=88.858, \sigma_{9}=18.646\end{array}$ & Estimated \\
\hline
\end{tabular}

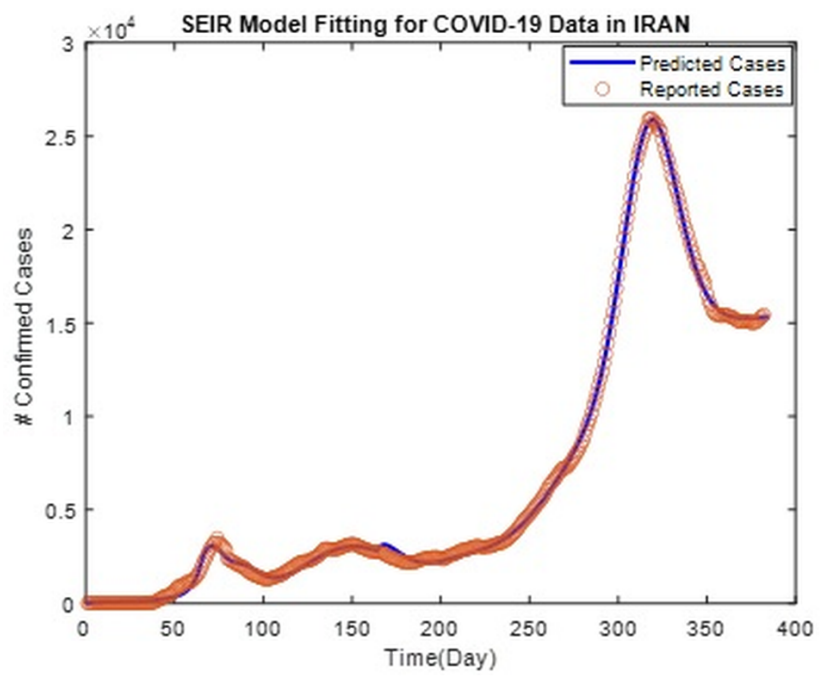

Figure 3. SEIR model fitting with daily notified cases of COVID-19 in Iran. The red circles represent the daily notified COVID-19 cases (in the time window $1 / 22 / 2020-2 / 07 / 2021$ ) and the solid blue line is the predicted trends with calibrated SEIR model.

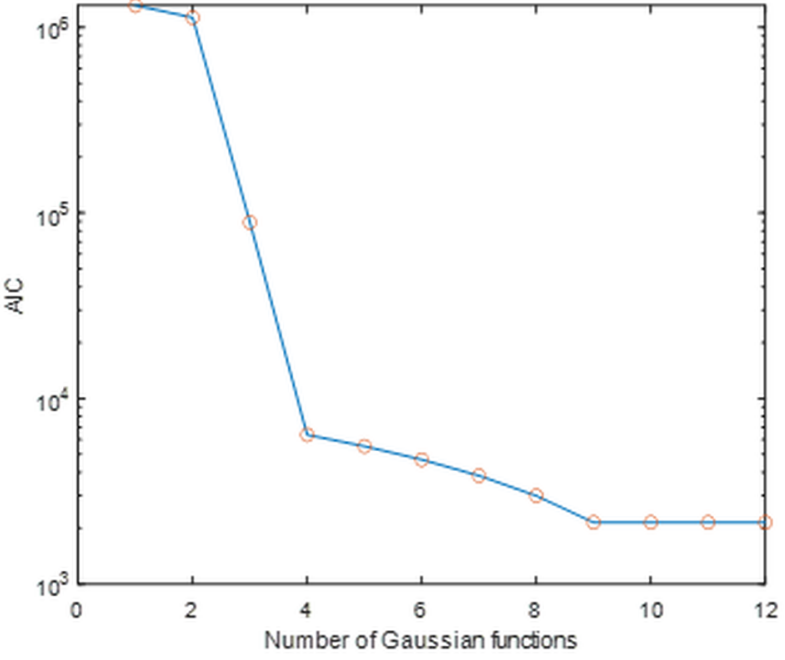

Figure 4. Trend of AIC in different settings of Gaussian functions. The minimum value of AIC and the simplest form of the model is created for nine Gaussian functions.
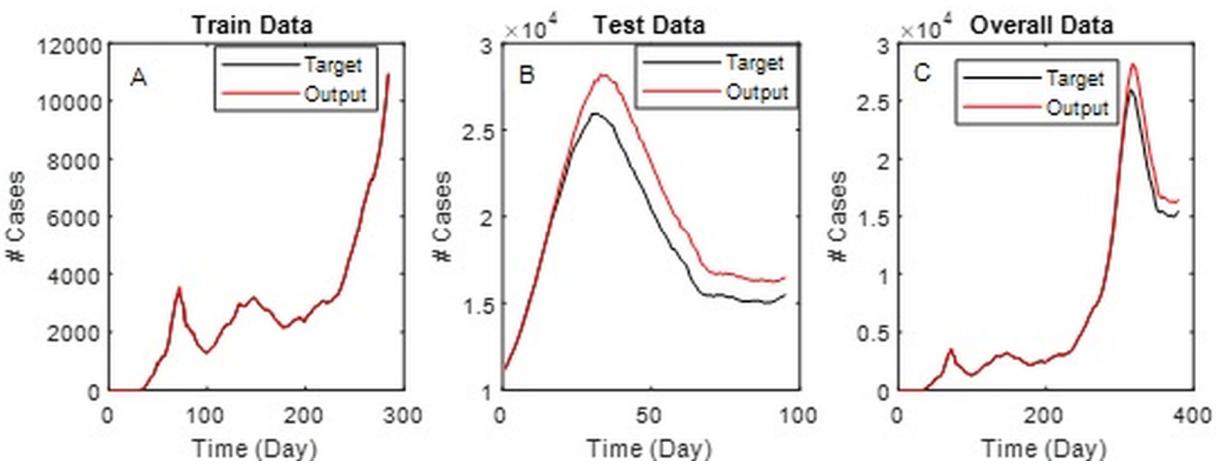

Figure 5. ANFIS model training and validation. The black line (Target) depicts the daily notified COVID-19 cases in Iran and red lines (Output) depict ANFIS model results in the time window 1/22/2020 - 11/11/2020 (panel A for model training), and in the time window 11/12/2020- 02/07/2021 (panel B for model validation), and time window 1/22/2020 - 2/07/2021 (panel C for overall data). 

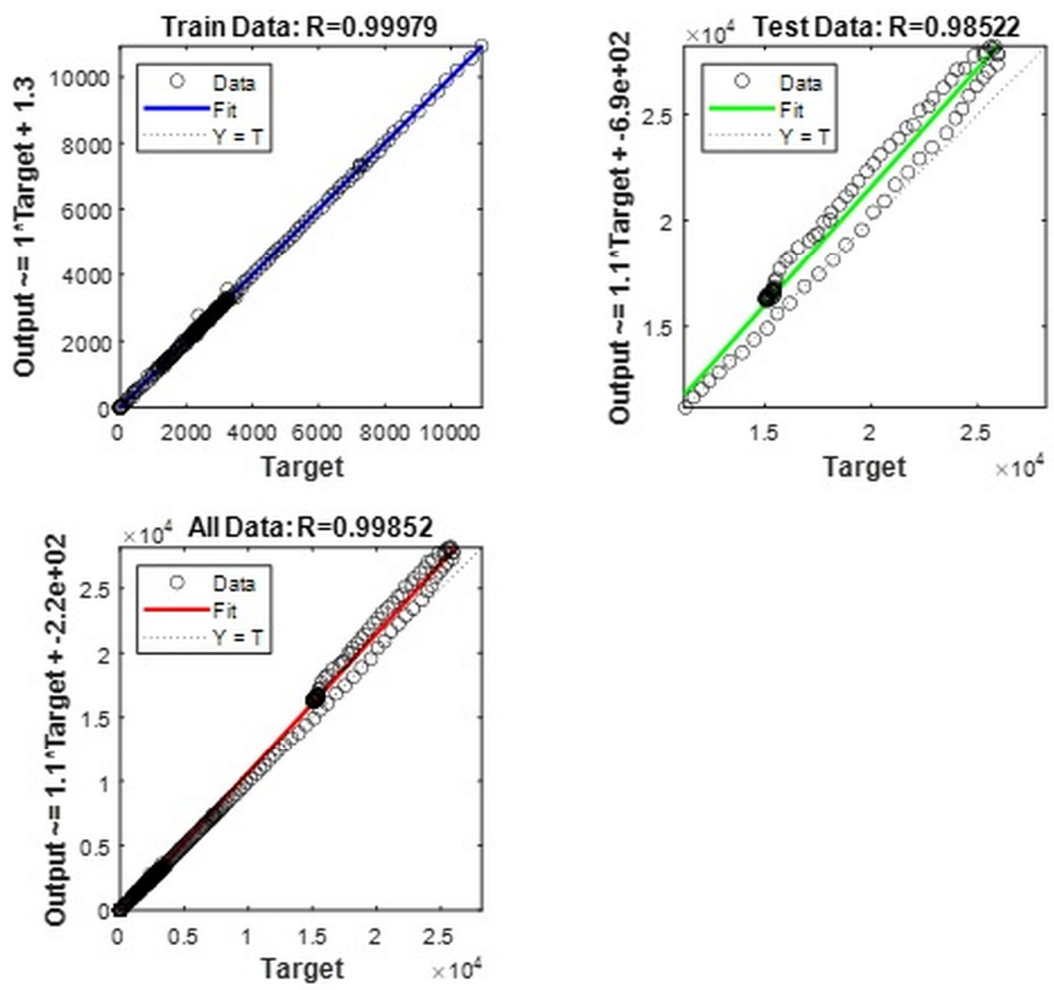

Figure 6. Regression of ANFIS model results. Comparison between COVID-19 cases predicted by ANFIS model and data in different training, validation, and overall data set are shown in different panels.

A

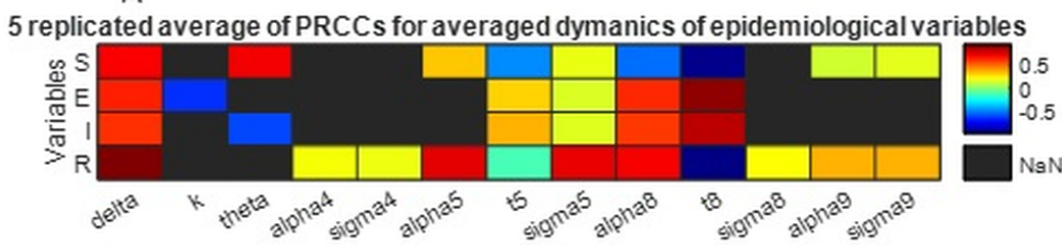

Parameters

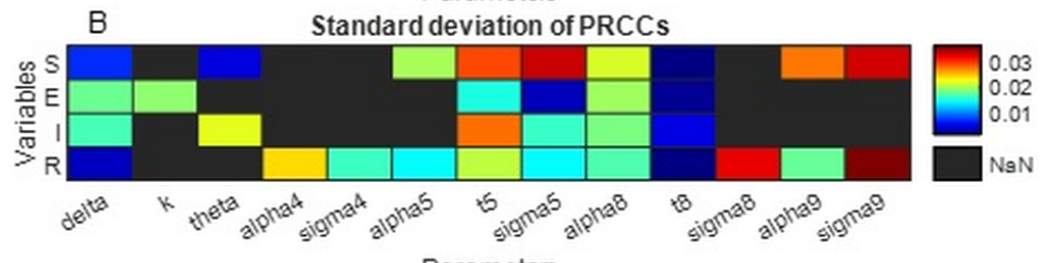

Parameters

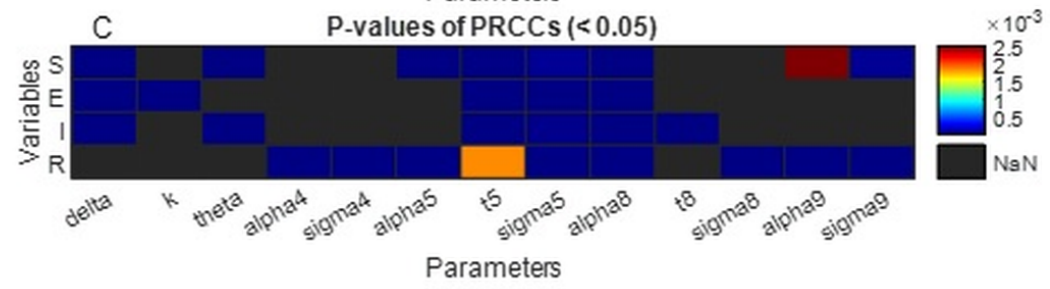

Figure 7. Global Sensitivity Analysis. (A) Five replicated average of statistically significant PRCC values (p-value<0.05) for susceptible, exposed, infected, and recovered populations. (B) The standard deviation of PRCC values for five replications of PRCC analysis (1000 run) and $(\mathrm{C})$ corresponding p-values. Black pixels (' $\mathrm{NaN}$ ') show 'not a number' and represent no significant correlation between the population of states (that are depicted in the vertical axis) and model parameters (elements in the horizontal axis).

\section{Results of PRCC analysis}

The five replicated averages of PRCC values are shown in Figure 7. A, and corresponding standard deviations (for 5 replications of PRCC analysis) and corresponding significant $\mathrm{p}$ values depicted in Figures 7b, and 7c, respectively. As depicted in Figure 7a, there is a negative correlation between the averaged population of infected individuals and parameter $\Theta$ (Probability that an infected patient will be recovered). Also, the averaged population of susceptible, exposed, infected, and recovered compartments have a strong positive correlation with parameter $\delta$ (average recruitment rate). 


\section{Model assessment in two scenarios}

We evaluated the ANFIS model in two different scenarios including social distancing and not distancing to predict the trend of COVID-19 new cases for the future six months. We implemented the scenarios of social distancing and non-social distancing by changing $( \pm 25 \%)$ the value of the average recruitment rate. In social distancing and non-distancing scenarios, we set $\delta=15$ and $\delta=25$, respectively. As shown in Figure 8, our simulations in the social distancing scenario revealed that the trend of COVID-19 new cases (red lines of left panel) for the future six months is oscillating and converge to a steady state. In this scenario, the third big peak of COVID-19 cases will not repeat, and the population of new COVID-19 cases will converge slightly to lower populations that will decrease as the vaccine is injected into the population. The right panels of Figure 8 show the fuzzy membership functions of input $1: X(t-2)$, input $2: X(t-1)$, input $3: X(t)$ of the ANFIS model. The $X$-axis of these panels describes the population of COVID-19 cases at times $t-2, t-1, t$, and the Y-axis of these panels depicts the $\alpha$-cut levels of fuzzy membership functions. As depicted in the right panels, ten membership functions are devoted to the inputs $X(t-2), X(t-1), X(t)$ to predict the population of COVID-19 new cases $X(t+1)$. The results of the lack of social distancing scenario are shown in Figure 9. As shown in the left panel of this Figure, the lack of social distancing may cause the recurrence of the third peak and the population of COVID-19 new cases in Iran as a fluctuating function will constantly decrease and subsequently increase. As shown in the right panel of this Figure, we devoted two membership functions for input 1: $X(t-2)$, input 2: $X(t-1)$, and input 3: $X(t)$ of the ANFIS model.

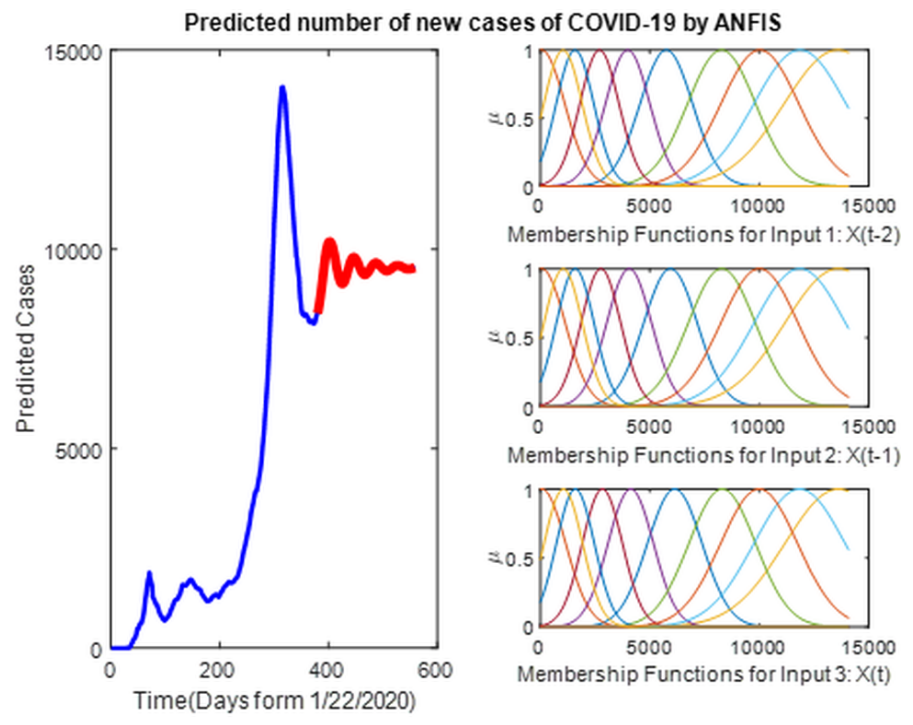

Figure 8. ANFIS model assessment by assuming social distancing scenario. The trend of COVID-19 cases trained (blue line in the left panel) and predicted (red line in the left panel) by ANFIS model and fuzzy membership function of inputs 1, 2, and 3 of the model (right panels).

\section{Discussion}

Given the many challenges posed by the SARS-CoV-2 coronavirus, predicting the course of COVID-19 disease plays an important role in government policies for the future. The widespread of the SARS-CoV-2 coronavirus in all the provinces of Iran has made it a difficult task to control, and fight the COVID-19 outbreak. Because Iran is economically weak and is under sanctions, the government was unable to support the people for quarantine, and the people carried out their economic activities by participating in society. The control of the corona crisis in this condition is a very challenging task. In this study, we presented a new parametrized SEIR model with epidemiological data of Iran and combine it with the ANFIS model as a time series predictive method. Since the trend of the COVID-19 cases in Iran is oscillating, it is difficult to predict its trend using SEIR modeling. In this study, we evaluated calibrated SEIR model under different scenarios of social distancing to capture the dynamics of COVID-19 cases. Then, we used these dynamics to train an ANFIS model to predict new COVID-19 cases by this rigorous time series prediction approach in different scenarios. We could have used the SEIR model to predict the number of new COVID-19 cases by assigning new Gaussian functions to the infection transmission rate function $(\beta)$, but in this case, a more complex model would be obtained. The dynamics of the SEIR model depend on function $\beta$. As shown by PRCC analysis, the population of infected patients strongly correlated with parameters of function $\beta$ and any change in this function affected the predicted dynamics of new infected cases.

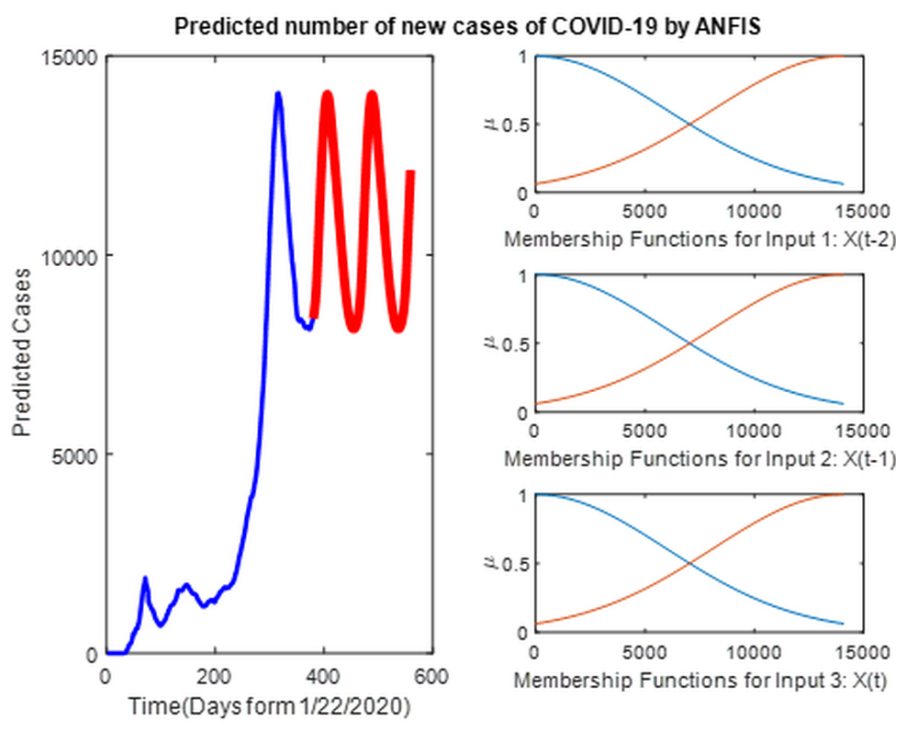

Figure 9. ANFIS model assessment by assuming social and nondistancing scenario. The trend of COVID-19 cases trained (blue line in the left panel) and predicted (red line in the left panel) by ANFIS model and fuzzy membership function of inputs 1, 2, and 3 of the model (right panels). 
Using the ANFIS model, we avoided complicating the SEIR model and made accurate and reliable predictions. We evaluated our trained ANFIS model under two different scenarios including social distancing and lack of social distancing to assess the effect of distancing on the COVID-19 outbreak. We know that the first case of COVID-19 in Iran was reported on February 19, 2020, and then the announced cases had an increasing trend until 10 days after Nowruz 2020 (fist peak of population of COVID-19 cases). After that, with the implementation of quarantine policies, the prevalence of COVID-19 decreased and again, as the severity of social distancing decreased, the likelihood of transmission of the virus increased (second peak). After the first and second peaks and with the outbreak of influenza, Iran suffered the third peak in the number of new people with COVID-19 disease. Flu leads to a weakened immune system and an increase in the number of people prone to the disease in the community.$^{20}$ On the other hand, increasing the number of referrals to medical centers due to flu leads to more people being exposed to the coronavirus and ultimately predicts an increase in the number of COVID-19 patients. Now (at the time of writing his manuscript), Iran is close to Nowruz 2021, and this crisis could lead to a fourth peak in the population of new COVID-19 cases.

Our six-month projections by SEIR and ANFIS model in Iran revealed that in a social distancing scenario, the population of new COVID-19 cases will decrease and will reach a steady-state that by applying vaccination the trend of COVID-19 new cases will reduce more. Also, our six-month projections in the not distancing scenario show that the population of new COVID-19 cases will oscillate and the third big peak will recur. Therefore, according to our predictions in the scenario of lack of social distancing, we will see the fourth and fifth peaks in the future six months.

Numerous studies have predicted the trend of COVID-19 disease in different countries. ${ }^{11,21-24}$ A study conducted by Syed \& Sibgatullah indicates that Pakistan could hit the peak number of infectious cases on May 26th, 2020 and by June 24th, 2020, $90 \%$ of the population will have become infected with the virus. $^{25}$ In another study conducted by Zareie et al. in Iran, predicted that 29000 people were infected from March 25 to April 15, 2020, and predicted that about 1715 people were infected at the highest point on March 25, 2020. Based on Zareie et al results, non-observance of health protocols by holding religious ceremonies in August 2020 eventually led to the increasing trend of the disease in the country. ${ }^{22}$ Sukumaran mentioned that the abolition of intercity traffic restrictions and non-compliance with health protocols have been among the reasons for the increase in the prevalence of the disease. ${ }^{26}$ Based on the results of our study, the evidence suggests that the abolition of intercity traffic restrictions during and after Nowruz 2021 and the reopening of public places can be one of the reasons for the increase in the prevalence of the disease. Numerous factors can increase the incidence of the disease. Failure to observe the social distance, holding religious ceremonies, and increased travel volume due to holidays are some reasons. ${ }^{22}$ A study conducted by Picchiotti showed that compliance with social distancing leads to reduce the COVID$19 .{ }^{11}$

In this study, for the first time, we combined SEIR modeling with ANFIS time series prediction approach. The infection transmission rate of our SEIR model is a function of time and this function helped us to follow the oscillating trend of COVID19 cases in Iran. Calibrated SEIR model by epidemic data of Iran was evaluated in different scenarios of social distancing. ANFIS model for the first time was used to analyze predicted dynamics of the SEIR model to extract optimized rules for the prediction of the COVID-19 trend in Iran. Although, our method was used on the data set of Iran, but it is not confined to this data and this method can be used to analyze the data set of all countries.

\section{Conclusion}

Up to Feb 7, 2021, the total number of confirmed COVID-19 cases and deaths in Iran are 1466563 and 57980, respectively. This rises with new confirmed cases every day reported from Iran. Currently, the COVID-19 vaccine in Iran is being injected into doctors, nurses, health workers, and hospital staff, and the country plans to vaccinate the entire population of the country by next year. In this condition, the only possible way to control the prevalence is social distancing to prevent the more susceptible population from being exposed and infected. In the present study, we designed an SEIR and ANFIS model that is parameterized by epidemic data made available by WHO and referring to the period 1/22/2020 - 2/07/2021. Reliable Predictions of the model can be used in guiding clinical and political decisions.

Since cultural factors such as the Nowruz holiday in 2020 in Iran affected the infection rate by SARS-CoV-2 coronavirus and caused the first peak in the number of COVID-19 patients. The second and third peaks in the number of infected people were due to the abolition of quarantine. At the time of writing this manuscript, Iranian society is close to Nowruz 2021, and there is a possibility of peak recurrences in the COVID-19 patient population. Our data analysis by parameterized SEIR and ANFIS model revealed that in the lack of social distancing scenario after the Nowruz holiday in 2021, the fourth and fifth big peaks of new COVID-19 cases will be seen.

\section{Code availability}

The MATLAB codes of model simulations are available by reasonable request from the corresponding author.

\section{Ethics approval}

This study was approved by the institutional review board of Qazvin University of Medical Sciences. 


\section{Acknowledgement}

We sincerely appreciate the efforts of doctors, nurses and all healthcare workers trying to keep us safe in the COVID-19 crisis.

\section{References}

1. Chen L, Liu W, Zhang Q, et al. RNA based mNGS approach identifies a novel human coronavirus from two individual pneumonia cases in 2019 Wuhan outbreak. Emerg Microbes Infect. 2020;9(1):313-319. https://doi.org/10.1080/22221751.2020.1725399

2. Wangping J, Ke H, Yang S, et al. Extended SIR prediction of the epidemics trend of COVID-19 in Italy and compared with Hunan, China. Front Med. 2020;7:169. https://doi.org/10.3389/fmed.2020.00169

3. Hamzah FAB, Lau C, Nazri H, Ligot D V, Lee G, Tan CL. CoronaTracker: worldwide COVID-19 outbreak data analysis and prediction. Bull World Heal Organ. 2020;1:32. https://doi.org/10.2471/BLT.20.255695

4. Roser M, Ritchie H, Ortiz-Ospina E, Hasell J. Coronavirus disease (COVID-19)-Statistics and research. Our World data. Published online 2020. https://ourworldindata.org/coronavirus

5. Chen N, Zhou M, Dong X, et al. Epidemiological and clinical characteristics of 99 cases of 2019 novel coronavirus pneumonia in Wuhan, China: a descriptive study. Lancet. 2020;395(10223):507-513. https://doi.org/10.1016/S0140-6736(20)30211-7

6. Huang C, Wang Y, Li X, et al. Clinical features of patients infected with 2019 novel coronavirus in Wuhan, China. Lancet. 2020;395(10223):497-506. https://doi.org/10.1016/S0140-6736(20)30183-5

7. Bao L, Deng W, Huang B, et al. The pathogenicity of SARS-CoV-2 in hACE2 transgenic mice. Nature. 2020;583:830-833. https://doi.org/10.1038/s41586-020-2312-y

8. Niazkar M, Niazkar HR. COVID-19 Outbreak: Application of Multi-gene Genetic Programming to Country-based Prediction Models. Electron J Gen Med. 2020;17(5). https://doi.org/10.29333/ejgm/8232

9. Wang P, Zheng X, Li J, Zhu B. Prediction of epidemic trends in COVID-19 with logistic model and machine learning technics. Chaos, Solitons \& Fractals. 2020;139:110058. https://doi.org/10.1016/j.chaos.2020.110058

10. Abdi M, Mirzaei R. Iran Without Mandatory Quarantine and with Social Distancing Strategy Against Coronavirus Disease (COVID19). Heal Secur. 2020;18(3). https://doi.org/10.1089/hs.2020.0041

11. Picchiotti N, Salvioli M, Zanardini E, Missale F. COVID-19 Italian and Europe epidemic evolution: A SEIR model with lockdowndependent transmission rate based on Chinese data. 2020. https://doi.org/10.2139/ssrn.3562452

12. Al-Qaness MAA, Fan H, Ewees AA, Yousri D, Abd Elaziz M. Improved ANFIS model for forecasting Wuhan City Air Quality and analysis COVID-19 lockdown impacts on air quality. Environ Res. 2021;194:110607. https://doi.org/10.1016/j.envres.2020.110607

13. Behnood A, Golafshani EM, Hosseini SM. Determinants of the infection rate of the COVID-19 in the US using ANFIS and virus optimization algorithm (VOA). Chaos, Solitons \& Fractals. 2020;139:110051. https://doi.org/10.1016/j.chaos.2020.110051

14. Saif S, Das P, Biswas S. A Hybrid Model based on mBA-ANFIS for COVID-19 Confirmed Cases Prediction and Forecast. J Inst Eng Ser B. 2021:1-14. https://doi.org/10.1007/s40031-021-00538-0

15. Hu, S.Akaike Information Criterion; Center for Research in Scientific Computation, North Carolina State University: Raleigh, NC,USA, 2007.

16. Marino S, Hogue IB, Ray CJ, Kirschner DE. A methodology for performing global uncertainty and sensitivity analysis in systems biology. J Theor Biol. 2008;254(1):178-196. https://doi.org/10.1016/j.jtbi.2008.04.011

17. WHO. Life Expectancy in Iran. Published 2020. Accessed August 30, 2020. https://www.worldlifeexpectancy.com/iran-lifeexpectancy

18. Sardar T, Nadim SS, Rana S, Chattopadhyay J. Assessment of Lockdown Effect in Some States and Overall India: A Predictive Mathematical Study on COVID-19 Outbreak. Chaos, Solitons \& Fractals. 2020;139:110078. https://doi.org/10.1016/j.chaos.2020.110078

19. Zhang J, Litvinova M, Wang W, et al. Evolving epidemiology and transmission dynamics of coronavirus disease 2019 outside Hubei province, China: a descriptive and modelling study. Lancet Infect Dis.2020;20(7):793-802. https://doi.org/10.1016/S14733099(20)30230-9

20. Hsih W-H, Cheng M-Y, Ho M-W, et al. Featuring COVID-19 cases via screening symptomatic patients with epidemiologic link during flu season in a medical center of central Taiwan. J Microbiol Immunol Infect. 2020;53(3):459-466. https://doi.org/10.1016/j.jmii.2020.03.008

21. Abdulmajeed K, Adeleke M, Popoola L. Online forecasting of COVID-19 cases in Nigeria using limited data. Data Br. 2020;30:105683. https://doi.org/10.1016/j.dib.2020.105683 
22. Zareie B, Roshani A, Mansournia MA, Rasouli MA, Moradi G. A model for COVID-19 prediction in Iran based on China parameters. medRxiv. 2020. https://doi.org/10.1101/2020.03.19.20038950

23. Sun J, Chen X, Zhang Z, et al. Forecasting the long-term trend of COVID-19 epidemic using a dynamic model. Sci Rep. 2020;10(1):110. https://doi.org/10.1038/s41598-020-78084-w

24. Fanelli D, Piazza F. Analysis and forecast of COVID-19 spreading in China, Italy and France. Chaos, Solitons \& Fractals. 2020;134:109761. https://doi.org/10.1016/j.chaos.2020.109761

25. Syed F, Sibgatullah S. Estimation of the Final Size of the COVID-19 Epidemic in Pakistan. medRxiv. 2020. https://doi.org/10.1101/2020.04.01.20050369

26. Sukumaran R, Patwa P, Sethuraman T V, et al. COVID-19 Outbreak Prediction and Analysis using Self Reported Symptoms. arXiv Prepr. arXiv:210110266. 2020. 